\title{
Isolated congenital complete heart block in a five-year-old seronegative girl born to a woman seropositive for human immunodeficiency virus: a case report
}

Pedro Pallangyo ${ }^{1 *}$, Isaac Mawenya ${ }^{1}$, Paulina Nicholaus ${ }^{1}$, Henry Mayala' ${ }^{1}$ Amida Kalombola², Godwin Sharau ${ }^{3}$, Naiz Majani ${ }^{3}$ and Mohamed Janabi ${ }^{1}$

\begin{abstract}
Background: Congenital complete heart block is a life-threatening condition which is highly associated with autoimmune and connective tissue disorders. Presence of maternal autoantibodies for associated conditions increases the risk of delivering a child with congenital complete heart block, however, less than a half of all women with such antibodies are symptomatic even after delivery. Mortality rate is highest during the neonatal period (45\%) and about two-thirds of all cases will require permanent pacing at some point in their lives.

Case presentation: We report a case of isolated complete heart block in a 5-year-old HIV-free girl of African descent born to an HIV-infected woman with no prior history of autoimmune disorders. She was referred to us with chief complaints of recurrent syncopal attacks and effort intolerance since birth. A physical examination was unremarkable except for her being small for her age (body mass index $16.3 \mathrm{~kg} / \mathrm{m}^{2}$ ) and bradycardia. Her vital signs were within acceptable range with the exception of her pulse rate, which ranged between 22 and 34 beats/minute. An echocardiogram revealed a sinus bradycardia, otherwise a structurally normal heart. An electrocardiogram showed atrioventricular dissociation in keeping with third-degree atrioventricular block. The child underwent a permanent epicardial pacemaker insertion and has been symptom-free following pacing.
\end{abstract}

Conclusions: Despite its infrequency and life-threatening potential, patients with congenital complete heart block have an excellent survival rate with timely diagnosis and intervention. An incidental detection of bradycardia in a fetus during routine obstetrical ultrasound examination should increase the index of suspicion for congenital complete heart block and warrant a screening for associated maternal autoantibodies.

Keywords: Congenital complete heart block, Congenital third-degree AV block, Isolated congenital heart block, Recurrent syncopal attacks, Symptomatic bradycardia, Case report

\section{Background}

Congenital complete heart block $(\mathrm{CCHB})$ is a rare, permanent, irreversible and life-threatening anomaly that is present in about 1 of every 20,000 live-born infants [1]. It may be diagnosed in utero, during infanthood or early childhood and is frequently associated with maternal autoantibodies, or rarely with a congenital structural deformity of the heart

\footnotetext{
* Correspondence: pedro.pallangyo@gmail.com

${ }^{1}$ Department of Cardiovascular Medicine, The Jakaya Kikwete Cardiac

Institute, P.O Box 65141, Dar es Salaam, Tanzania

Full list of author information is available at the end of the article
}

[2]. The risk of delivering a child with CCHB is higher among women who are anti-SSA/Ro or anti-SSB/La positive and in those with autoimmune hypothyroidism [3]. The transplacental transfer of maternal autoantibodies to $\mathrm{SSA} / \mathrm{Ro}$ or SSB/La ribonucleoproteins may lead to inflammation and ultimately fibrosis of the fetal conduction system [4]. Interestingly, over $50 \%$ of mothers carrying such autoantibodies are asymptomatic and are identified only after they have children with a heart block [4]. Furthermore, the risk of CCHB recurrence among women with associated autoantibodies rises to $17 \%$ in the second offspring 
and may rise up to $50 \%$ in subsequent deliveries [4]. For instance, infants with neonatal lupus erythromatosus (NLE) have up to a $30 \%$ risk of developing $\mathrm{CCHB}$ compared to infants without it and such infants comprise over $90 \%$ of all CCHB cases [5, 6].

Clinically, $\mathrm{CCHB}$ presents with recurrent fainting attacks, a slow pulse rate and electrocardiographic (ECG) findings in keeping with complete heart block $[1,2,4]$. About twothirds of all CCHB cases will require permanent pacing, which provides an excellent prognosis especially among those with the isolated form [7]. Overall mortality rate in $\mathrm{CCHB}$ is about $20 \%$, it is higher in utero (27\%) or during early infancy (45\%) and is associated with delayed pacing therapy $[7,8]$. Comparatively, CCHB cases with comorbid cardiac structural malformations have the worst prognosis [2]. We report a case of isolated complete heart block in a 5 -year-old girl with a history of recurrent syncopal attacks and effort intolerance since birth.

\section{Case presentation}

A 5-year-old HIV-free girl of African descent was referred to us with chief complaints of recurrent syncopal attacks and effort intolerance since birth. She is a third-born child to a 38-year-old, single, HIV-infected woman. Her mother first gave birth at the age of 27, she was HIV-free then but the baby was born with spina bifida and anencephaly and survived for just a few hours after birth. She then gave birth to a baby girl 2 years later; the child is healthy and alive until today. She became pregnant again 4 years after the birth of her second child. During an antenatal clinic visit for this pregnancy, she tested negative for hepatitis B and her syphilis screening was nonreactive. She was never screened for rubella or autoimmune conditions. She had low hemoglobin concentration throughout (9.4-10.9 g/dL), but received hematenics, antihelminthics, antimalarial and tetanus toxoid immunization as per protocol. She was diagnosed with HIV infection during the antenatal clinic visit at 24 weeks' gestation and was started on antiretroviral medication. She reported that she took the medication for 3 days only and decided to stop because she did not want her family to know of her HIV serostatus. She had severe hyperemesis gravidarum during her first trimester and was hospitalized seven times due to this. Her pregnancy thereafter was uneventful and she gave birth to a full-term baby girl weighing 2.3 kilograms who scored 7/10 on the APGAR scale. The child was given nevirapine at birth and she was instructed to continue giving it for 6 weeks, but for the same reasons she stopped her child's nevirapine on the third day. She exclusively breastfed her child for 6 months and never resumed her or her child's antiretroviral medication during this period. The mother denied any symptoms suggestive of hypothyroidism, connective tissue or autoimmune disorders before, during, and after pregnancy.
During her third child's first few weeks of life, the mother observed that she was breastfeeding poorly and was sleeping for longer duration compared to her sister at her age. At about 8 weeks, it was observed that the child would go into an unarousable state on exertion or while breastfeeding. This state lasted between 30 minutes and 1 hour, and upon regaining consciousness, the child was lethargic. The child never presented with a crying episode or convulsions prior to her loss of consciousness. The child was taken to a health facility and the mother was told that her child's heart was beating slower than usual but was assured that it would go away with time. She continued to present with syncopal attacks until the age of 2 years but was never admitted for this or any other illness and had normal developmental milestones. She was free of such episodes for about 3 years, however, she was mostly lethargic with a decreased effort capacity, had a poor weight gain and was generally inactive for her age. Three weeks prior to this index admission, she presented again with episodes of loss of consciousness which persisted until she came to our attention. She had experienced between three and five episodes per day, each lasting for about an hour and after regaining consciousness she slept for up to 12 hours. This time round, minor activities including swallowing and rising from a lying position led to syncope. Since birth, the child had never had features of heart failure or a transient rash suggestive of neonatal lupus erythromatosus. Furthermore, the mother denies any prescription of steroids for herself or her child between pregnancy and now.

Upon admission to our institute, the child underwent a thorough physical examination and a series of investigations. The physical examination was unremarkable except for her being small for her age (body mass index (BMI) $16.3 \mathrm{~kg} / \mathrm{m}^{2}$ ) and bradycardia. Her vital signs were within acceptable range with the exception of her pulse rate, which ranged between 22 and 34 beats/minute. She had normal electrolytes, renal and liver function, full blood count and random blood sugar test results. Serology results for HIV and hepatitis (B and C) were negative. An echocardiogram revealed a sinus bradycardia, otherwise a structurally normal heart. An ECG showed AV dissociation in keeping with thirddegree atrioventricular (AV) block, Fig. 1. Anti-SSA/Ro and anti-SSB/La antibodies tests are unavailable in the country and were not screened for, however, the mother tested negative for anti-double-stranded deoxyribonucleic acid (dsDNA), antinuclear antibodies (ANA) and antineutrophil cytoplasmic antibodies (ANCA) autoantibodies. The child underwent a permanent epicardial pacemaker insertion and has been symptomfree following pacing. 


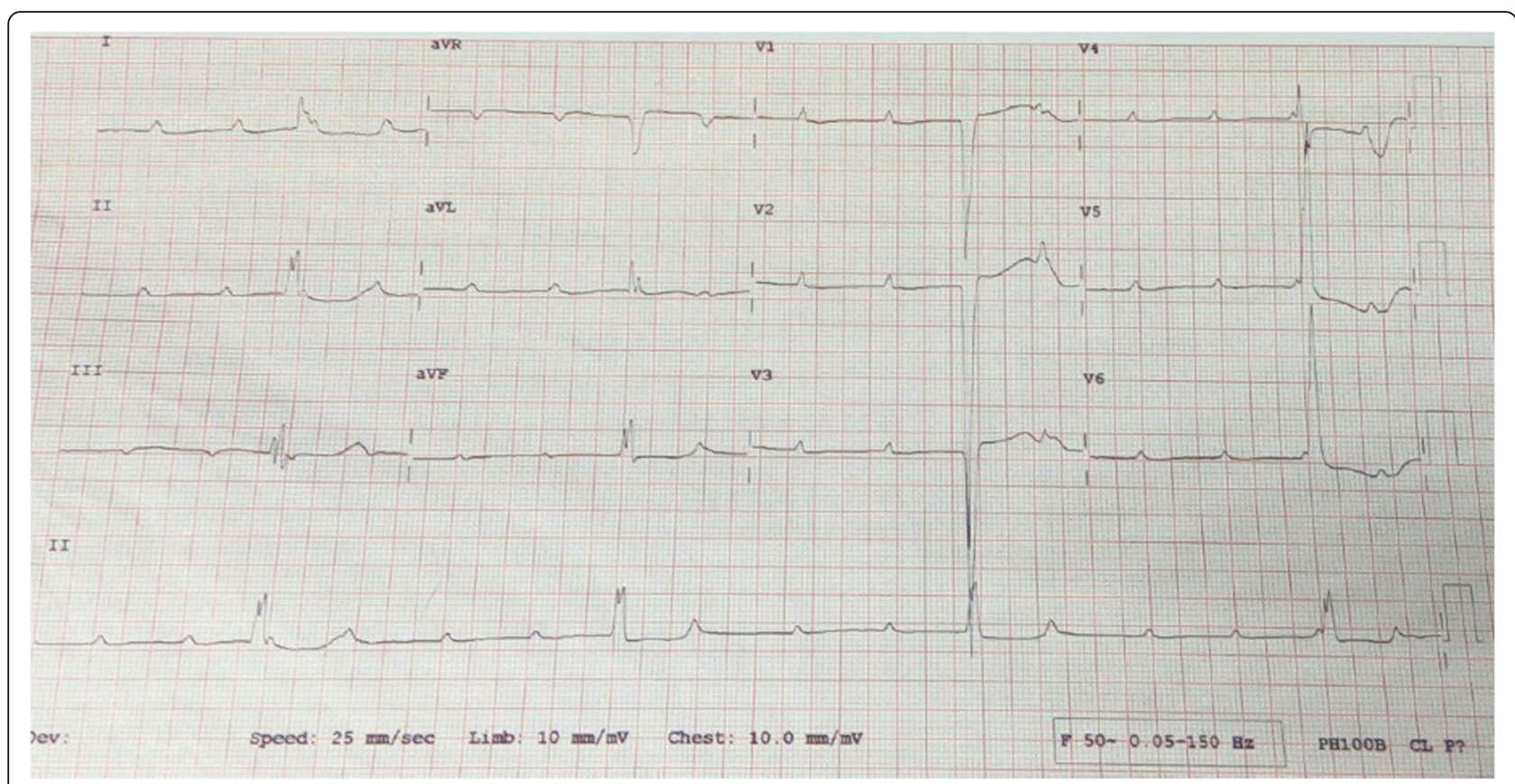

Fig. 1 Electrocardiogram showing atrioventricular dissociation in keeping with the diagnosis of complete heart block

\section{Discussion}

Complete congenital heart block is a potentially fatal condition although in this modern era the prognosis is excellent. In the developed world, the majority of patients are diagnosed in utero, however, in resourcelimited settings where fetal echocardiography is a rare practice, diagnosis is exclusively made during infancy [9]. Early diagnosis of isolated CCHB, which is highly associated with autoimmune disorders, remains a challenge even in the best centers because the majority of women are asymptomatic even after delivery $[1,4,5,8]$. When CCHB is diagnosed in utero in a woman with autoantibodies, several therapeutic approaches including use of steroids, sympathomimetics, plasmapheresis, and fetal pacing have been implicated [10]. Children with CCHB exhibiting symptoms early in life are regarded high risk and necessitate urgent pacing. A pacemaker aims to prevent sudden cardiac death, provide symptomatic relief, and improve the quality of life [2].

In the case presented, the child was symptomatic from birth but a definitive diagnosis of CCHB was made at around the age of 5 . While mortality rate due to $\mathrm{CCHB}$ is reportedly high during the neonatal period, our case is very fortunate to have survived until age 5 without any intervention despite the presence of recurrent syncopal attacks since birth. This case, despite undergoing all the diagnostic hurdles, served as a learning platform to practitioners in our center as this was the first-ever case of $\mathrm{CCHB}$ to be diagnosed, paced, and documented in Tanzania.

\section{Conclusions}

In conclusion, despite of its infrequency and lifethreatening potential, patients with $\mathrm{CCHB}$ have an excellent survival rate with timely diagnosis and intervention. Furthermore, among women with established associated autoimmune conditions, frequent echocardiographic fetal heart rate assessment and necessary in utero interventions are essential for better fetal outcomes. On the other hand, incidental detection of bradycardia in a fetus during routine obstetrical ultrasound examination should increase an index of suspicion for $\mathrm{CCHB}$ and warrant a screening for associated maternal autoantibodies.

\section{Abbreviations}

ANA: Antinuclear antibodies; ANCA: Antineutrophil cytoplasmic

antibodies; AV: Atrioventricular; CCHB: Complete congenital heart block; dsDNA: Double-stranded deoxyribonucleic acid; ECG: Electrocardiogram; NLE: Neonatal lupus erythromatosus

\section{Acknowledgements}

The authors are grateful to the pediatricians and surgeons from the Jakaya Kikwete Cardiac Institute. Furthermore, the authors are indebted to the pediatric department of the Muhimbili National Hospital for their continual cooperation in improving children's health.

\section{Funding}

Not applicable.

\section{Availability of data and material}

Not applicable.

\section{Authors' contributions}

PP and IM took the history and performed the physical examination. NM performed the echocardiography. GS performed the pacemaker insertion. PP wrote the initial draft of the manuscript. All authors reviewed and 
contributed to the final version of this case report. All authors read and approved the final manuscript.

\section{Competing interests}

The authors declare that they have no competing interests.

\section{Consent for publication}

Written informed consent was obtained from the patient's legal guardians for publication of this case report and any accompanying images. A copy of the written consent is available for review by the Editor-in-Chief of this journal.

\section{Ethics approval and consent to participate}

Ethical clearance was sought from the Directorate of Research of the Jakaya Kikwete Cardiac Institute.

\section{Author details}

'Department of Cardiovascular Medicine, The Jakaya Kikwete Cardiac Institute, P.O Box 65141, Dar es Salaam, Tanzania. ${ }^{2}$ Department of Pediatric and Child Health, Muhimbili National Hospital, P.O Box 65000, Dar es Salaam, Tanzania. ${ }^{3}$ Department of Pediatric Cardiology, The Jakaya Kikwete Cardiac Institute, P.O Box 65141, Dar es Salaam, Tanzania.

Received: 15 August 2016 Accepted: 29 September 2016

Published online: 19 October 2016

\section{References}

1. Massa AC, Freitas I, Borges A, Serrano F. Congenital complete atrioventricular block - case report and review of the literature. Acta Obstet Ginecol Port. 2015;9(4):318-21

2. Kiblawi M, Naeem A, Al Attrash E, Kar S, Goud BK. Complete congenital heart block in a newborn associated with maternal systemic lupus erythematosus: a case report. Int J Med Students. 2013;1(3):128-31.

3. Hon KL, Leung AK. Neonatal lupus erythematosus. Autoimmune Dis. 2012;2012:301274

4. Yildirim A, Tunaodlu FS, Karaadac AT. Neonatal congenital heart block. Indian Pediatr. 2013;50(5):483-8.

5. Izmirly PM, Saxena A, Kim MY, Wang D, Sahl SK, Llanos C, et al. Maternal and fetal factors associated with mortality and morbidity in a multi-racial/ethnic registry of anti-SSA/Ro-associated cardiac neonatal lupus. Circulation. 2011;124(18):1927-35.

6. Rein AJJT, Mevorach D, Perles Z, et al. Early diagnosis and treatment of atrioventricular block in the fetus exposed to maternal anti-SSA/Ro-SSB/La antibodies: a prospective, observational, fetal kinetocardiogram-based study. Circulation. 2009;119:1867-72.

7. Moak JP, Barron KS, Hougen TJ, et al. Congenital heart block: development of late-onset cardiomyopathy, a previously underappreciated sequela. JACC. 2001;37(1):238-42.

8. Eronen M, Siren MK, Ekblad $\mathrm{H}$, et al. Short-and long-term outcome of children with congenital complete heart block diagnosed in utero or as a newborn. Pediatrics. 2000;106:86-91.

9. Waltuck J, Buyon JP. Autoantibody associated complete heart block: outcome in mothers and children. Ann Intern Med. 1994;120:544-51.

10. Buyon JP, Waltuck J, Kleinman C, Copel J. In utero identification and therapy of congenital heart block. Lupus. 1995;4(2):116-21.

\section{Submit your next manuscript to BioMed Central and we will help you at every step:}

- We accept pre-submission inquiries

- Our selector tool helps you to find the most relevant journal

- We provide round the clock customer support

- Convenient online submission

- Thorough peer review

- Inclusion in PubMed and all major indexing services

- Maximum visibility for your research

Submit your manuscript at www.biomedcentral.com/submit
C Biomed Central 